\title{
On Improving the Tradeoff between Symbol Rate and Diversity Gain Using Quasi-Orthogonal Space-Time Block Codes with Linear Receivers
}

\author{
Kazuyuki MORIOKA $^{\dagger \text { a) }}$, Student Member and David ASANO ${ }^{\dagger \dagger}$, Member $^{2}$
}

SUMMARY In this letter, the tradeoff between symbol rate and diversity gain of Space-Time Block Codes (STBCs) with linear receivers is considered. It is known that Group Orthogonal-Toeplitz Codes (GOTCs) can achieve a good tradeoff with linear receivers. However, the symbol rate of GOTCs is limited to that of the base Orthogonal Space-Time Block Codes (OSTBCs). We propose to simply change the GOTC base codes from OSTBCs to Quasi-Orthogonal Space-Time Block Codes (Q-OSTBCs). QOSTBCs can improve the symbol rate of GOTCs at the expense of diversity gain. Simulation results show that Q-OSTBC based GOTCs can improve the tradeoff between symbol rate and diversity gain over that of the original GOTCs.

key words: Orthgonal Space-Time Block Codes, Quasi-Orthgonal SpaceTime Block Codes, Group Orthgonal-Toeplitz Codes

\section{Introduction}

Recently, Multi Input Multi Output (MIMO) systems have received much attention in mobile communication system research. MIMO systems can increase channel capacity by sending parallel data on multiple paths between the transmitter and receiver [1]. Transmitting data in parallel to increase channel capacity is called spatial multiplexing. On the other hand, spatial diversity achieves coding gain by sending the same data via multiple antennae and combining them at the receiver [2]-[5].

There are tradeoffs between spatial multiplexing and spatial diversity. Zheng and Tse [6] studied the optimal tradeoff between spatial multiplexing and spatial diversity and showed an optimal tradeoff curve. Also, Gamal extended Linear Dispersion (LD) codes [7] and proposed LAttice Space Time (LAST) codes [8] which can achieve an optimal tradeoff. However these techniques use a non-linear Maximum Likelihood (ML) receiver. In mobile terminals, linear receivers such as Zero-Forcing (ZF) and Minimum Mean Square Error (MMSE) are preferred because of battery life limitations. In this letter, we consider the tradeoff between symbol rate and diversity gain of Space Time Block Codes (STBCs) with linear receivers.

\footnotetext{
Manuscript received April 26, 2012.

Manuscript revised July 15, 2012.

${ }^{\dagger}$ The author is with the Department of Mathematics and System Development, The Interdisciplinary Graduate School of Science and Technology, Shinshu University, Nagano-shi, 380-8553 Japan.

${ }^{\dagger}$ The author is with the Department of Computer Science and Engineering, Shinshu University, Nagano-shi, 380-8553 Japan.

a) E-mail: Kazuyuki.Morioka@ gmail.com

DOI: 10.1587/transcom.E95.B.3763
}

Orthogonal Space-Time Block Codes (OSTBCs) were originally proposed by Alamouti [2] and extended to the general case by Tarokh et al. [3]-[5]. OSTBCs can achieve full diversity and can be decoded using maximum likelihood detection with linear receivers by using the orthogonality of the code. OSTBCs have such a good property but their symbol rate is limited to $(k+1) / 2 k$, where $2 k-1$ or $2 k$ represents the number of transmit antennae [9]. Also, OSTBC can achieve full rate and full diversity at the same time only when the number of transmit antennae is two [10].

So, STBCs which have a good tradeoff between symbol rate and diversity gain with linear receivers have received much attention [11]-[13]. Liu et al. [11] proposed Toeplitz Codes which improve the symbol rate of STBC with linear receivers. Shang and Xia [12] improved the symbol rate and diversity gain by combining Toeplitz Codes and Alamouti's OSTBC. Also, Wang et al. [13] extended their approach and proposed a construction method of STBCs combining Toeplitz Codes and general OSTBCs to improve the tradeoff. The codes proposed in [13] are called Group Orthgonal-Toeplitz Codes (GOTCs) including OSTBC [2][5], Toeplitz Codes [11], and Overlapped Alamouti Codes (OACs) [12] as special cases. However, the symbol rate of GOTCs is limited to that of the base OSTBCs which is used when it is generated. In this letter, we propose to simply change the GOTC base codes from OSTBCs to QuasiOrthogonal Space-Time Block Codes (Q-OSTBCs) [14] to improve the symbol rate of GOTCs.

This letter is organized as follows. In Sect. 2, we apply Quasi-Orthogonal Space-Time Block Codes [14] to GOTCs. Simulation results and some comments about the tradeoff between symbol rate and diversity gain are presented in Sect. 3. Finally, we conclude this letter in Sect. 4.

\section{Proposed Method}

The system model, assumptions, definitions and notations used here are the same as [13]. To construct GOTCs, $N_{t}$ transmit antennae are divided into $K$ groups, and the number in group $k$ is $n_{k}$. Hence, $\sum_{k=1}^{K} n_{k}=N_{t}$ is satisfied. Also, the $L$ transmitted symbols are divided into groups of $K^{\prime}$ symbols. Then, as mentioned in [13], the symbol rate of GOTCs is described as follows.

$$
R_{S}=\frac{L}{T_{o}\left(L / K^{\prime}+n_{1}-1\right)},
$$


where, $T_{o}$ is the length of the time slot of the base OSTBC, $O_{K^{\prime}, K}$. Here, $O_{K^{\prime}, K}$ represents an OSTBC which sends $K^{\prime}$ symbols using $K$ transmit antennae. From Eq. (1), $R_{S} \rightarrow K^{\prime} / T_{o}$ when $L$ is sufficiently large. This is the same as the symbol rate of the base OSTBC, $O_{K^{\prime}, K}$. Hence, the symbol rate of GOTCs is limited to the symbol rate of the base OSTBC. In this letter, we denote a GOTC as GOTC $\left(\mathcal{N}_{N_{t}, K}\right.$, $O_{K^{\prime}, K}$ ) when its grouping scheme is $\mathcal{N}_{N_{t}, K}$ and its base OSTBC is $O_{K^{\prime}, K}$. For example, to divide $N_{t}=8$ transmit antennae into $K=4$ groups, we can use grouping schemes such that $\mathcal{N}_{8,4}=(2,2,2,2), \mathcal{N}_{8,4}=(1,2,2,3)$ and so on. The detailed construction method of GOTCs is omitted here for want of space, but is described in the original paper [13].

The main idea of this letter is to replace the base OSTBCs with Quasi-Orthogonal Space-Time Block Codes (Q-OSTBCs) to improve the symbol rate of the original GOTCs. Q-OSTBCs can improve the symbol rate at the expense of the orthogonality of the code [14]. It is pointed out in [13] that Q-OSTBC also has Group Orthogonality but it cannot achieve full diversity. However, the possibility to improve the tradeoff at the expense of diversity gain is not considered in [13]. The purpose of this letter is to improve the symbol rate using Q-OSTBCs at the expense of diversity gain and determine the parameters which achieve a better tradeoff. In this letter, we represent Q-OSTBCs which transmit $K^{\prime}$ symbols with $K$ transmit antennae as $Q_{K^{\prime}, K}$. We also represent GOTCs generated by $Q_{K^{\prime}, K}$ as $\operatorname{GOTC}\left(\mathcal{N}_{N_{t}, K}\right.$, $\left.Q_{K^{\prime}, K}\right)$. A Q-OSTBC which transmits 4 symbols in 4 time slots with 4 transmit antennae is described as follows [14].

$$
Q_{4,4}=\left[\begin{array}{cccc}
x_{1} & x_{2} & x_{3} & x_{4} \\
-x_{2}^{*} & x_{1}^{*} & -x_{4}^{*} & x_{3}^{*} \\
-x_{3}^{*} & -x_{4}^{*} & x_{1}^{*} & x_{2}^{*} \\
x_{4} & -x_{3} & -x_{2} & x_{1}
\end{array}\right] .
$$

Then, $\bar{X}_{\mathcal{N}_{N_{t}, 4}}=\operatorname{GOTC}\left(\mathcal{N}_{N_{t}, 4}, Q_{4,4}\right)$ becomes as follows instead of Eq. (11) in [13].

$$
\bar{X}_{\mathcal{N}_{N_{t}, 4}}=\left[\begin{array}{cccc}
\boldsymbol{S}_{r, 1}^{(1)} & \boldsymbol{S}_{r, 2}^{(2)} & \boldsymbol{S}_{r, 3}^{(3)} & \boldsymbol{S}_{r, 4}^{(4)} \\
-\tilde{\boldsymbol{S}}_{r, 1}^{(2) *} & \tilde{\boldsymbol{S}}_{r, 2}^{(1) *} & -\tilde{\boldsymbol{S}}_{r, 3}^{(4) *} & \tilde{\boldsymbol{S}}_{r, 4}^{(3) *} \\
-\tilde{\boldsymbol{S}}_{r, 1}^{(3) *} & -\tilde{\boldsymbol{S}}_{r, 2}^{(4) *} & \tilde{\boldsymbol{S}}_{r, 3}^{(1) *} & \tilde{\boldsymbol{S}}_{r, 4}^{(2) *} \\
\boldsymbol{S}_{r, 1}^{(4)} & -\boldsymbol{S}_{r, 2}^{(3)} & -\boldsymbol{S}_{r, 3}^{(2)} & \boldsymbol{S}_{r, 4}^{(1)}
\end{array}\right] .
$$

Futhermore, when the grouping scheme is $\mathcal{N}_{8,4}=(2,2,2,2)$, the code becomes

$$
\bar{X}_{\mathcal{N}_{8,4}}=\left[\begin{array}{cc|cc|cc|cc}
s_{1} & 0 & s_{3} & 0 & s_{5} & 0 & s_{7} & 0 \\
s_{2} & s_{1} & s_{4} & s_{3} & s_{6} & s_{5} & s_{8} & s_{7} \\
0 & s_{2} & 0 & s_{4} & 0 & s_{6} & 0 & s_{8} \\
\hline-s_{4}^{*} & 0 & s_{2}^{*} & 0 & -s_{8}^{*} & 0 & s_{6}^{*} & 0 \\
-s_{3}^{*} & -s_{4}^{*} & s_{1}^{*} & s_{2}^{*} & -s_{7}^{*} & -s_{8}^{*} & s_{5}^{*} & s_{6}^{*} \\
0 & -s_{3}^{*} & 0 & s_{1}^{*} & 0 & -s_{7}^{*} & 0 & s_{5}^{*} \\
\hline-s_{6}^{*} & 0 & -s_{8}^{*} & 0 & s_{2}^{*} & 0 & s_{4}^{*} & 0 \\
-s_{5}^{*} & -s_{6}^{*} & -s_{7}^{*} & -s_{8}^{*} & s_{1}^{*} & s_{2}^{*} & s_{3}^{*} & s_{4}^{*} \\
0 & -s_{5}^{*} & 0 & -s_{7}^{*} & 0 & -s_{1}^{*} & 0 & s_{3}^{*} \\
\hline s_{7} & 0 & -s_{5} & 0 & -s_{3} & 0 & s_{1} & 0 \\
s_{8} & s_{7} & -s_{6} & -s_{5} & -s_{4} & -s_{3} & s_{2} & s_{1} \\
0 & s_{8} & 0 & -s_{6} & 0 & -s_{4} & 0 & s_{2}
\end{array}\right] .
$$

Table 1 Comparison of code rate when $L$ is 24, 48 and 72.

\begin{tabular}{c|c|c|c}
$L$ & 24 & 48 & 72 \\
\hline \hline Toeplitz [11] & 0.774 & 0.872 & 0.911 \\
\hline OAC [12] & 0.800 & 0.888 & 0.923 \\
\hline GOTC $\left(\mathcal{N}_{8,4}, O_{3,4}\right)[13]$ & 0.666 & 0.705 & 0.720 \\
\hline $\operatorname{GOTC}\left(\mathcal{N}_{8,4}, Q_{4,4}\right)$ & 0.857 & 0.923 & 0.947
\end{tabular}

The symbol rate of $\operatorname{GOTC}\left(\mathcal{N}_{N_{t}, K}, Q_{K^{\prime}, K}\right)$ can be described as Eq. (1) which is the same as that of $\operatorname{GOTC}\left(\mathcal{N}_{N_{t}, K}, O_{K^{\prime}, K}\right)$. So,if $L$ is the same, the symbol rate of GOTC $\left(\mathcal{N}_{N_{t}, K}, Q_{K^{\prime}, K}\right)$ becomes higher than that of GOTC $\left(\mathcal{N}_{N_{t}, K}, O_{K^{\prime}, K}\right)$, because $K^{\prime}$ is larger than that of $O_{K^{\prime}, K}$. Similarly, $\operatorname{GOTC}\left(\mathcal{N}_{N_{t}, K}\right.$, $\left.Q_{K^{\prime}, K}\right)$ needs a smaller value of $L$ to achieve the same symbol rate as $\operatorname{GOTC}\left(\mathcal{N}_{N_{t}, K}, O_{K^{\prime}, K}\right)$. This means the decoding delay is small and it has tolerance to channel conditions. A comparison of the symbol rate for $O_{3,4}, Q_{4,4}$, Toeplitz Codes [11], and Overlapped Alamouti Codes (OAC) [12] are described in Table 1 . Table 1 shows that a higher symbol rate can be achieved if we use Q-OSTBC as the base code. However, we must consider the loss in diversity gain caused by improving the symbol rate, because there is a tradeoff between symbol rate and diversity gain. In the next section, we evaluate the performance for the same frequency efficiency by computer simulations and show that GOTCs with Q-OSTBCs can improve the symbol rate without much loss in performance.

\section{Simulation Results}

In this section, we compare the performance of QOSTBC based GOTCs with original GOTCs. We use $\operatorname{GOTC}\left(\mathcal{N}_{N_{t}, 2}, \mathrm{O}_{2,2}\right)$ instead of OAC, because OAC and $\operatorname{GOTC}\left(\mathcal{N}_{N_{t}, 2}, \mathrm{O}_{2,2}\right)$ are essentially the same as mentioned in [13]. We use Alamouti [2] codes as $O_{2,2}$ and we use Eqs. (7) and (8) in [13] as $O_{3,3}$ and $O_{3,4}$ respectively. Also, we use Eq. (2) in this letter as $Q_{4,4}$. A systematic approach to construct OSTBC with an arbitrary number of transmit antennas is described in [9]. We use $B_{8}^{\prime}$ in [9] as OSTBC with 8 transmit antennae. To evaluate fairly, we compare the performance under the same frequency efficiency, and we consider both low and high frequency efficiency cases. We also assume a quasi-flat fading environment and use gray coding. Finally, a Zero Forcing $(\mathrm{ZF})$ receiver is used as the linear receiver.

Figure 1 shows the bit error probability when the number of transmit antennae and receive antennae are 8 and 1 respectively, and the frequency efficiency is $2.5 \mathrm{bit} / \mathrm{s} / \mathrm{Hz}$. Also, Fig. 2 shows the result when the number of transmit and receive antennae are the same as above, and the frequency efficiency is $5.0 \mathrm{bit} / \mathrm{s} / \mathrm{Hz}$. The parameters used here are shown in Table 2. For example, the frequency efficiency of OSTBC with 16QAM is $2.5 \mathrm{bit} / \mathrm{s} / \mathrm{Hz}$ because the symbol rate of OSTBC is 0.625 from Table 2 and 16QAM has 4 bits/symbol. So, the frequency efficiency is $4 \times 0.625=2.5 \mathrm{bit} / \mathrm{s} / \mathrm{Hz}$. We represent OSTBC based 


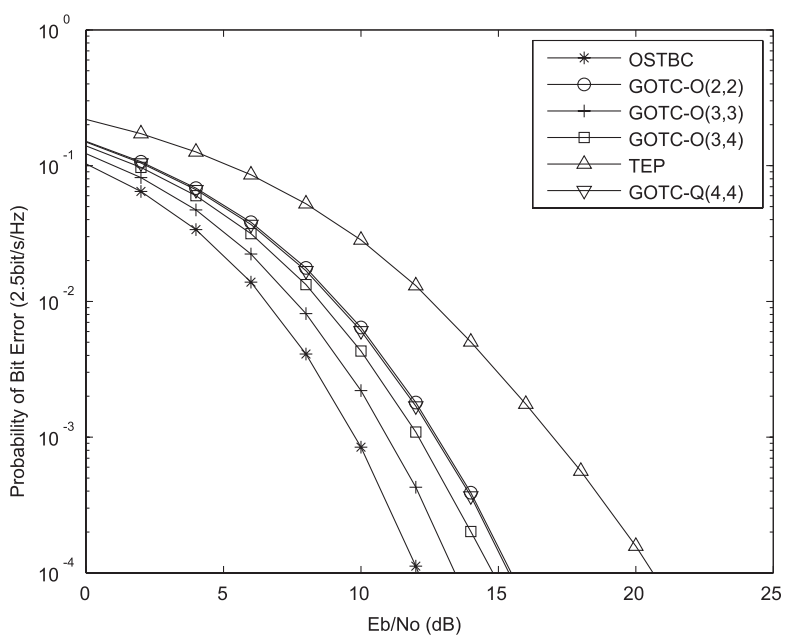

Fig. 1 An average bit error rate comparison of the proposed QuasiOSTBC based GOTCs (GOTC-Q) with OSTBC based GOTCs [13], OSTBC [3] and Toeplitz code [11] at an efficiency of $2.5 \mathrm{bit} / \mathrm{s} / \mathrm{Hz}$. The number of $\mathrm{Tx} / \mathrm{Rx}$ antennae are 8 and 1 respectively.

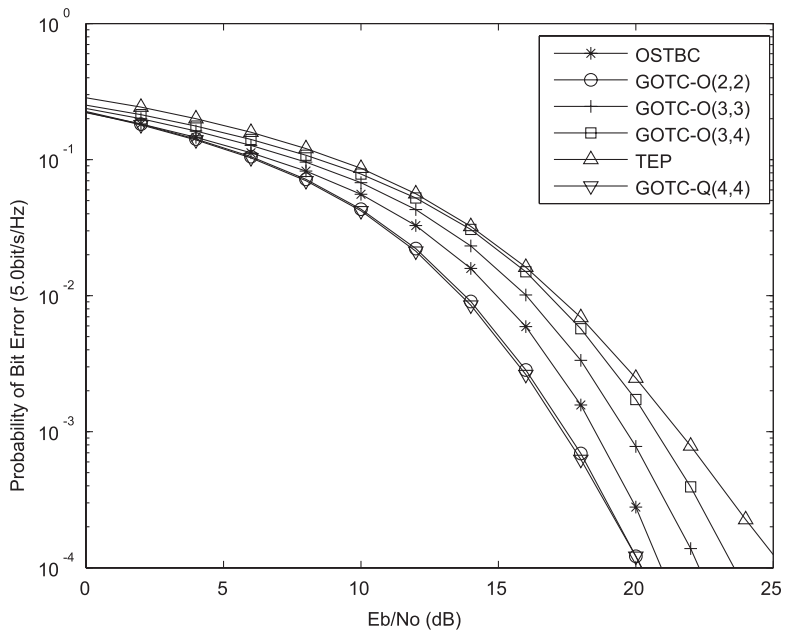

Fig. 2 An average bit error rate comparison of the proposed QuasiOSTBC based GOTCs (GOTC-Q) with OSTBC based GOTCs [13], OSTBC [3] and Toeplitz code [11] at an efficiency of $5.0 \mathrm{bit} / \mathrm{s} / \mathrm{Hz}$. The number of Tx/Rx antennae are 8 and 1 respectively.

Table 2 Simulation parameters when the frequency efficiency is $2.5 \mathrm{bit} / \mathrm{s} / \mathrm{Hz}$ and $5.0 \mathrm{bit} / \mathrm{s} / \mathrm{Hz}$

\begin{tabular}{c|c|c|c|c} 
& $L$ & Rate & $2.5 \mathrm{bit} / \mathrm{s} / \mathrm{Hz}$ & $5.0 \mathrm{bit} / \mathrm{s} / \mathrm{Hz}$ \\
\hline \hline OSTBC [3] & - & 0.625 & 16QAM & 256QAM \\
\hline Toeplitz [11] & 35 & 0.833 & 8QAM & 64QAM \\
\hline GOTC $\left(\mathcal{N}_{8,2}, O_{2,2}\right)[13]$ & 30 & 0.833 & 8QAM & 64QAM \\
\hline $\operatorname{GOTC}\left(\mathcal{N}_{8,3}, O_{3,3}\right)[13]$ & 30 & 0.625 & 16QAM & 256QAM \\
\hline $\operatorname{GOTC}\left(\mathcal{N}_{8,4}, O_{3,4}\right)[13]$ & 15 & 0.625 & 16QAM & 256QAM \\
\hline $\operatorname{GOTC}\left(\mathcal{N}_{8,4}, Q_{4,4}\right)$ & 20 & 0.833 & 8QAM & 64QAM
\end{tabular}

$\operatorname{GOTC}\left(\mathcal{N}_{N_{t}, K}, O_{K^{\prime}, K}\right)$ [13] as GOTC-O $\left(K^{\prime}, K\right)$, and the QOSTBC based GOTC $\left(\mathcal{N}_{N_{t}, K}, Q_{K^{\prime}, K}\right)$ scheme proposed in this letter as GOTC-Q $\left(K^{\prime}, K\right)$ for short in the figures.

Figure 1 shows that the performance of $\operatorname{GOTC}\left(\mathcal{N}_{8,4}\right.$,
Table 3 Simulation parameters for various frequency efficiencies.

\begin{tabular}{c|c|c|c|c|c} 
& \multicolumn{4}{|c|}{$L$} & Modulation \\
\hline \hline Toeplitz $[11]$ & 14 & 21 & 35 & 77 & $8 \mathrm{QAM} / 64 \mathrm{QAM}$ \\
\hline $\operatorname{GOTC}\left(\mathcal{N}_{8,2}, O_{2,2}\right)[13]$ & 12 & 18 & 30 & 66 & $8 \mathrm{QAM} / 64 \mathrm{Q} A M$ \\
\hline $\operatorname{GOTC}\left(\mathcal{N}_{8,3}, O_{3,3}\right)[13]$ & 12 & 18 & 30 & 66 & $16 \mathrm{QAM} / 256 \mathrm{QAM}$ \\
\hline $\operatorname{GOTC}\left(\mathcal{N}_{8,4}, O_{3,4}\right)[13]$ & 6 & 9 & 15 & 33 & $16 \mathrm{QAM} / 256 \mathrm{QAM}$ \\
\hline $\operatorname{GOTC}\left(\mathcal{N}_{8,4}, Q_{4,4}\right)$ & 8 & 12 & 20 & 44 & $8 \mathrm{QAM} / 64 \mathrm{QAM}$
\end{tabular}

$\left.O_{3,4}\right)$ outperforms that of $\operatorname{GOTC}\left(\mathcal{N}_{8,4}, Q_{4,4}\right)$ when the frequency efficiency is low $(2.5 \mathrm{bit} / \mathrm{s} / \mathrm{Hz})$. From Table 2, to achieve a frequency efficiency of $2.5 \mathrm{bit} / \mathrm{s} / \mathrm{Hz}$ with $\operatorname{GOTC}\left(\mathcal{N}_{8,4}, O_{3,4}\right)$, 16QAM modulation must be used. On the other hand, for $\operatorname{GOTC}\left(\mathcal{N}_{8,4}, Q_{4,4}\right), 8 \mathrm{QAM}$ modulation is sufficient to achieve the same $2.5 \mathrm{bit} / \mathrm{s} / \mathrm{Hz}$. Generally, the difference in power efficiency between 8QAM and 16QAM is about $2 \mathrm{~dB}$ (e.g. Figure 8.13 in [15]). In this case, $\operatorname{GOTC}\left(\mathcal{N}_{8,4}, O_{3,4}\right)$ has better performance, because the loss in diversity gain caused by improving the symbol rate has a larger effect on the performance than the improvement in power efficiency caused by using 8QAM rather than 16QAM. OSTBC has the best performance because the diversity gain has a larger effect on the performance in the low frequency efficiency case.

On the other hand, Fig. 2 shows that the performance of $\operatorname{GOTC}\left(\mathcal{N}_{8,4}, Q_{4,4}\right)$ outperforms that of $\operatorname{GOTC}\left(N_{8,4}, O_{3,4}\right)$ when the frequency efficiency is high $(5.0 \mathrm{bit} / \mathrm{s} / \mathrm{Hz})$. Similarly, Table 2 shows that 256QAM modulation must be used to achieve a frequency efficiency of $5.0 \mathrm{bit} / \mathrm{s} / \mathrm{Hz}$ with $\operatorname{GOTC}\left(\mathcal{N}_{8,4}, O_{3,4}\right)$. On the other hand, for $\operatorname{GOTC}\left(\mathcal{N}_{8,4}, Q_{4,4}\right)$, 64QAM modulation is sufficient to achieve the same $5.0 \mathrm{bit} / \mathrm{s} / \mathrm{Hz}$. Generally, the difference in power efficiency between 64QAM and 256QAM is about $5 \mathrm{~dB}$ (e.g. Figure 8.13 in [15]). In this case, $\operatorname{GOTC}\left(\mathcal{N}_{8,4}, Q_{4,4}\right)$ has better performance, because the improvement in power efficiency caused by using 64QAM instead of 256QAM has a larger effect than the loss in diversity gain caused by improving the symbol rate.

Also, $\operatorname{GOTC}\left(\mathcal{N}_{8,4}, Q_{4,4}\right)$ has almost the same performance as $\operatorname{GOTC}\left(\mathcal{N}_{8,2}, O_{2,2}\right)$ in both low and high frequency efficiency cases. This is because Q-OSTBC is generated by combining $\operatorname{OSTBC}\left(O_{2,2}\right)$ [14]. However, Fig. 1 and 2 show that $\operatorname{GOTC}\left(\mathcal{N}_{8,4}, Q_{4,4}\right)$ has slightly better performance. For example, $\operatorname{GOTC}\left(\mathcal{N}_{8,4}, Q_{4,4}\right)$ is superior by $0.13 \mathrm{~dB}$ than $\operatorname{GOTC}\left(\mathcal{N}_{8,2}, O_{2,2}\right)$ to achieve BER $=10^{-3}$ when the frequency efficiency is $5.0 \mathrm{bit} / \mathrm{s} / \mathrm{Hz}$. This is because a larger diversity gain can be obtained when $Q_{4,4}$ is combined with $O_{2,2}$ and a smaller $L$, instead of $O_{2,2}$ with a larger $L$. Also, Table 2 shows that the value of $L$ required to achieve the same symbol rate is smaller when $\operatorname{GOTC}\left(\mathcal{N}_{8,4}, Q_{4,4}\right)$ is used instead of $\operatorname{GOTC}\left(\mathcal{N}_{8,2}, O_{2,2}\right)$. This means that the decoding delay is smaller when $\operatorname{GOTC}\left(\mathcal{N}_{8,4}, Q_{4,4}\right)$ is used instead of $\operatorname{GOTC}\left(\mathcal{N}_{8,2}, O_{2,2}\right)$.

Figure 3 shows the relationship between frequency efficiency and the SNR which achieves a bit error rate of $10^{-3}$. Here, we varied $L$, and we evaluated various frequency ef- 


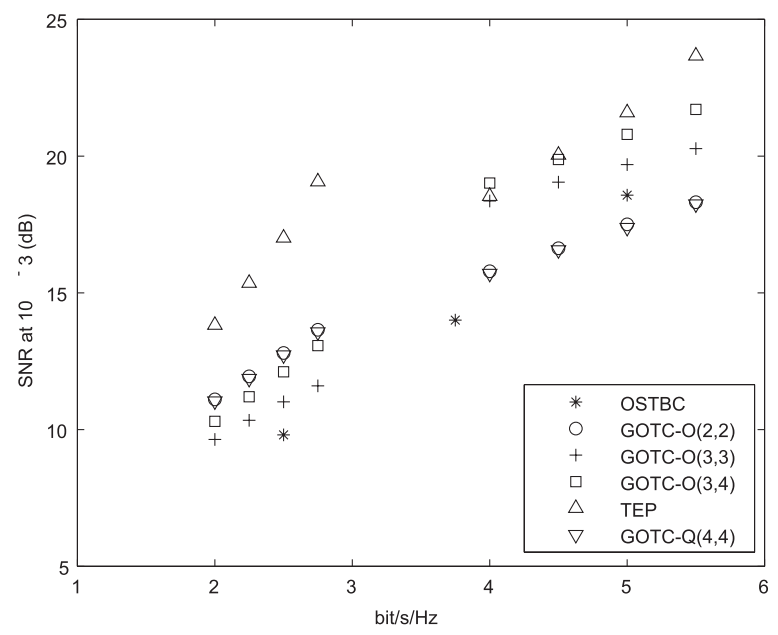

Fig. 3 An SNR level comparison of the proposed Quasi-OSTBC based GOTCs (GOTC-Q) with OSTBC based GOTCs [13], OSTBC [3] and Toeplitz code [11] to achieve an average bit error rate of $10^{-3}$. The number of $\mathrm{Tx} / \mathrm{Rx}$ antennae are 8 and 1 respectively.

ficiencies: $2.0,2.25,2.5,2.75 \mathrm{bit} / \mathrm{s} / \mathrm{Hz}$, and $4.0,4.5,5.0$, $5.5 \mathrm{bit} / \mathrm{s} / \mathrm{Hz}$. The values of $L$ used here are shown in Table 3. Also, we used $B_{8}^{\prime}$ in [9] as the OSTBC with 8 transmit antennae. This has the highest symbol rate $(0.625)$ for 8 transmit antennae [9]. We could not find other OSTBCs with 8 transmit antennae in the literature. Furthermore, we used the commonly used modulation schemes 16QAM, 64QAM and 256QAM, so, the frequency efficiencies of the OSTBCs used here are $2.5 \mathrm{bit} / \mathrm{s} / \mathrm{Hz}(=0.625 \times 4 \mathrm{bits}), 3.75 \mathrm{bit} / \mathrm{s} / \mathrm{Hz}$ $(=0.625 \times 6$ bits $)$, and $5.0 \mathrm{bit} / \mathrm{s} / \mathrm{Hz}(=0.625 \times 8 \mathrm{bits})$, respectively. We could not find combinations of OSTBCs with 8 transmit antennae and modulation schemes to achieve 2.0, $2.25,2.75,4.0,4.5$, and $5.5 \mathrm{bit} / \mathrm{s} / \mathrm{Hz}$. Therefore, we show plots for only $2.5,3.75$ and $5.0 \mathrm{bit} / \mathrm{s} / \mathrm{Hz}$ as a reference.

Figure 3 shows that the highest frequency efficiency $2.5 \mathrm{bit} / \mathrm{s} / \mathrm{Hz}$ can be achieved when we use OSTBC at an SNR of $9 \mathrm{~dB}$. Also, the highest frequency efficiency $2.75 \mathrm{bit} / \mathrm{s} / \mathrm{Hz}$ can be achieved when we use $\operatorname{GOTC}\left(\mathcal{N}_{8,3}, O_{3,3}\right)$ at $11.6 \mathrm{~dB}$. Furthermore, $\operatorname{GOTC}\left(\mathcal{N}_{8,3}, Q_{4,4}\right)$ has the best performance $(5.5 \mathrm{bit} / \mathrm{s} / \mathrm{Hz})$ when the SNR is $18.2 \mathrm{~dB}$. In this way, an automatic grouping scheme configuration can be done by measuring the received SNR and selecting the parameters to achieve the highest frequency efficiency. Here, as mentioned in [13], note that OSTBCs correspond to GOTCs with grouping scheme $\mathcal{N}_{N_{t}, N_{t}}$ and $L=1$. Also, the OAC and Toeplitz codes correspond to GOTCs with $\mathcal{N}_{N_{t}, 2}$ and $\mathcal{N}_{N_{t}, 1}$ respectively.

Finally, Fig. 4 shows the results when the frequency efficiency is $5.0 \mathrm{bit} / \mathrm{s} / \mathrm{Hz}$ and the number of received antennae is changed. Fig. 4 shows that $\operatorname{GOTC}\left(\mathcal{N}_{8,4}, Q_{4,4}\right)$ is better than $\operatorname{GOTC}\left(N_{8,4}, O_{3,4}\right)$, and the difference becomes larger as the number of received antennae increase. This is because the loss in diversity gain caused by improving the symbol rate is complemented by using multiple antennae. The results show that a better tradeoff can be achieved by using $\operatorname{GOTC}\left(\mathcal{N}_{8,4}, Q_{4,4}\right)$ and multiple antennae.

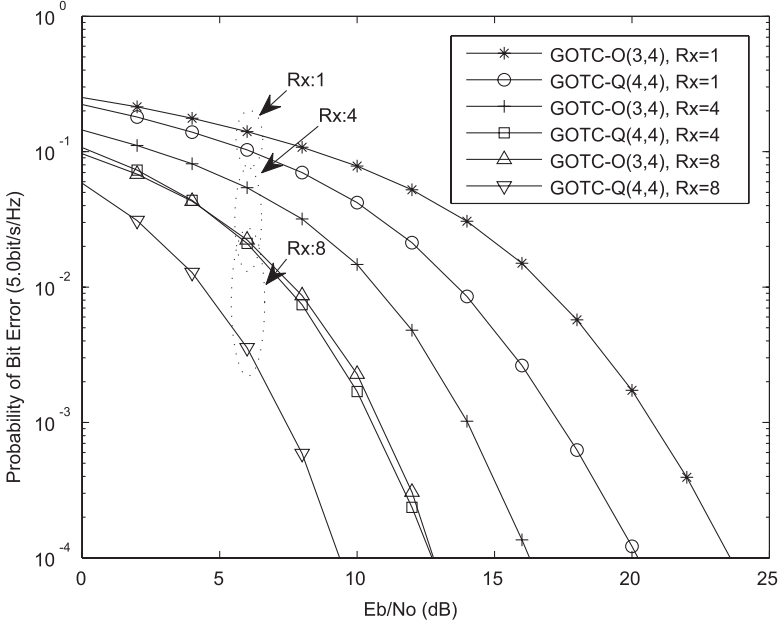

Fig. 4 An average bit error rate comparison of the proposed QuasiOSTBC based GOTCs (GOTC-Q) with OSTBC based GOTCs [13] at an efficiency of $5.0 \mathrm{bit} / \mathrm{s} / \mathrm{Hz}$. The number of Tx antennae is 8 and $\mathrm{Rx}$ antennae is 1,4 and 8 .

\section{Conclusions}

In this letter, the tradeoff between symbol rate and diversity gain of STBCs with linear receivers was considered. The results show that $\operatorname{GOTC}\left(\mathcal{N}_{8,4}, Q_{4,4}\right)$ has a better tradeoff than $\operatorname{GOTC}\left(\mathcal{N}_{8,4}, O_{3,4}\right)$. Also, $\operatorname{GOTC}\left(\mathcal{N}_{8,4}, Q_{4,4}\right)$ with a smaller value of $L$ has almost the same performance as $\operatorname{GOTC}\left(\mathcal{N}_{8,2}, O_{2,2}\right)$ with a larger value of $\mathrm{L}$. This means that the decoding delay is smaller for $\operatorname{GOTC}\left(\mathcal{N}_{8,4}, Q_{4,4}\right)$ than for $\operatorname{GOTC}\left(\mathcal{N}_{8,2}, O_{2,2}\right)$. In the future, we will work on the optimal GOTC base code to achieve the best tradeoff with linear receivers.

\section{References}

[1] E. Telatar, "Capacity of multi-antenna Gaussian channels," European Transactions on Telecommunications, vol.10, no.6, pp.585$595,1999$.

[2] S.M. Alamouti, "A simple transmit diversity technique for wireless communications," IEEE J. Sel. Areas Commun., vol.16, no.8, pp.1451-1458, 1998.

[3] V. Tarokh, N. Seshadri, and A. Calderbank, "Space-time codes for high data rate wireless communication: Performance criterion and code construction," IEEE Trans. Inf. Theory, vol.44, no.2, pp.744765,1998

[4] V. Tarokh, H. Jafarkhani, and A. Calderbank, "Space-time block codes from orthogonal designs," IEEE Trans. Inf. Theory, vol.45, no.5, pp.1456-1467, 1999

[5] V. Tarokh, H. Jafarkhani, and A. Calderbank, "Space-time block coding for wireless communications: Performance results," IEEE J. Sel. Areas Commun., vol.17, no.3, pp.451-460, 1999.

[6] L. Zheng and D. Tse, "Diversity and multiplexing: A fundamental tradeoff in multiple-antenna channels," IEEE Trans. Inf. Theory, vol.49, no.5, p.1073, 2003.

[7] B. Hassibi and B. Hochwald, "High-rate codes that are linear in space and time," IEEE Trans. Inf. Theory, vol.48, no.7, pp.1804 $1824,2002$.

[8] H. El Gamal, G. Caire, and M. Damen, "Lattice coding and decoding achieve the optimal diversity-vs-multiplexing tradeoff of MIMO 
channels," IEEE Trans. Inf. Theory, vol.50, no.6, pp.968-985, 2004

[9] K. Lu, S. Fu, and X. Xia, "Closed-form designs of complex orthogonal space-time block codes of rates $(\mathrm{k}+1) /(2 \mathrm{k})$ for $2 \mathrm{k}-1$ or $2 \mathrm{k}$ transmit antennas," IEEE Trans. Inf. Theory, vol.51, no.12, pp.43404347, 2005.

[10] X. Liang and X. Xia, "On the nonexistence of rate-one generalized complex orthogonal designs," IEEE Trans. Inf. Theory, vol.49, no.11, pp.2984-2988, 2003.

[11] J. Liu, J. Zhang, and K. Wong, "Full-diversity codes for MISO systems equipped with linear or ML detectors," IEEE Trans. Inf. Theory, vol.54, no.10, pp.4511-4527, 2008.
[12] Y. Shang and X. Xia, "Space-time block codes achieving full diversity with linear receivers," IEEE Trans. Inf. Theory, vol.54, no.10, pp.4528-4547, 2008.

[13] H. Wang, X. Xia, Q. Yin, and B. Li, "A family of space-time block codes achieving full diversity with linear receivers," IEEE Trans. Commun., vol.57, no.12, pp.3607-3617, 2009.

[14] H. Jafarkhani, "A quasi-orthogonal space time block code," IEEE Trans. Commun., vol.49, no.1, pp.1-4, 2001.

[15] F. Xiong, F. Xiong, and F. Xiong, Digital modulation techniques, Artech House Norwood, MA, USA, 2000. 